\title{
A Scalable Virtual Circuit Routing Scheme for ATM Networks*
}

\author{
Cengiz Alaettinoğlu \\ Information Sciences Institute \\ University of Southern California \\ Marina del Rey, CA 90292
}

\author{
Ibrahim Matta A. Udaya Shankar \\ Institute for Advanced Computer Studies \\ Department of Computer Science \\ University of Maryland \\ College Park, MD 20742
}

October 1994

\begin{abstract}
High-speed networks, such as ATM networks, are expected to support diverse quality-ofservice (QoS) requirements, including real-time QoS. Real-time QoS is required by many applications such as voice and video. To support such service, routing protocols based on the Virtual Circuit (VC) model have been proposed. However, these protocols do not scale well to large networks in terms of storage and communication overhead.

In this paper, we present a scalable VC routing protocol. It is based on the recently proposed viewserver hierarchy, where each viewserver maintains a partial view of the network. By querying these viewservers, a source can obtain a merged view that contains a path to the destination. The source then sends a request packet over this path to setup a real-time VC through resource reservations. The request is blocked if the setup fails. We compare our protocol to a simple approach using simulation. Under this simple approach, a source maintains a full view of the network. In addition to the savings in storage, our results indicate that our protocol performs close to or better than the simple approach in terms of VC carried load and blocking probability over a wide range of real-time workload.
\end{abstract}

Categories and Subject Descriptors: C.2.1 [Computer-Communication Networks]: Network Architecture and Design-packet networks; store and forward networks; C.2.2 [Computer-Communication Networks]: Network Protocols—protocol architecture; C.2.m [Routing Protocols]; F.2.m [Computer Network Routing Protocols].

\footnotetext{
* This work is supported in part by ARPA and Philips Labs under contract DASG60-92-0055 to Department of Computer Science, University of Maryland, and by National Science Foundation Grant No. NCR 89-04590. The work of C. Alaettinoğlu is also supported by National Science Foundation Grant No. NCR 93-21043. The views, opinions, and/or findings contained in this report are those of the author(s) and should not be interpreted as representing the official policies, either expressed or implied, of the Advanced Research Projects Agency, PL, the National Science Foundation, or the U.S. Government.
} 


\section{Contents}

1 Introduction $\quad 1$

2 Related Work 4

3 Viewserver Hierarchy Query Protocol $\quad 5$

4 Update Protocol for Dynamic Network Conditions 10

5 Evaluation $\quad 13$

6 Numerical Results $\quad 16$

6.1 Results for Network $1 \ldots \ldots \ldots \ldots \ldots \ldots$

6.2 Results for Network $2 \ldots \ldots \ldots \ldots \ldots$

7 Conclusions $r$ 


\section{Introduction}

Integrated services packet-switched networks, such as Asynchronous Transfer Mode (ATM) networks [21], are expected to carry a wide variety of applications with heterogeneous quality of service $(\mathrm{Q} O S)$ requirements. For this purpose, new resource allocation algorithms and protocols have been proposed, including link scheduling, admission control, and routing. Link scheduling defines how the link bandwidth is allocated among the different services. Admission control defines the criteria the network uses to decide whether to accept or reject a new incoming application. Routing concerns the selection of routes to be taken by application packets (or cells) to reach their destination. In this paper, we are mainly concerned with routing for real-time applications (e.g., voice, video) requiring QoS guarantees (e.g., bandwidth and delay guarantees).

To provide real-time QoS support, a number of virtual-circuit (VC) routing approaches have been proposed. A simple (or straightforward) approach to $\mathrm{VC}$ routing is the link-state full-view approach. Here, each end-system maintains a view of the whole network, i.e. a graph with a vertex for every node ${ }^{1}$ and an edge between two neighbor nodes. QoS information such as delay, bandwidth, and loss rate are attached to the vertices and the edges of the view. This QoS information is flooded regularly to all end-systems to update their views. When a new application requests service from the network, the source end-system uses its current view to select a source route to the destination end-system that is likely to support the application's requested QoS, i.e., a sequence of node ids starting from the source end-system and ending with the destination end-system. A VCsetup message is then sent over the selected source route to try to reserve the necessary resources (bandwidth, buffer space, service priority) and establish a VC.

Typically, at every node the VC-setup message visits, a set of admission control tests are performed to decide whether the new VC, if established, can be guaranteed its requested QoS without violating the QoS guaranteed to already established VCs. At any node, if these admission tests are passed, then resources are reserved and the VC-setup message is forwarded to the next node. On the other hand, if the admission tests fail, a VC-rejected message is sent back towards the source node releasing resource reservations made by the VC-setup message, and the application request is either blocked or another source route is selected and tried. If the final admission tests at the destination node are passed, then a VC-established message is sent back towards the source node confirming resource reservations made during the forward trip of the VC-setup message. Upon receiving the VC-established message, the application can start transmitting its packets over its

\footnotetext{
1 We refer to switches and end-systems collectively as nodes.
} 
reserved VC. This VC is torn down and resources are released at the end of the transmission.

Clearly, the above simple routing scheme does not scale up to large networks. The storage at each end-system and the communication cost are proportional to $N \times d$, where $N$ is the number of nodes and $d$ is the average number of neighbors to a node.

A traditional solution to this scaling problem is the area hierarchy used in routing protocols such as the Open Shortest Path First (OSPF) protocol [18]. The basic idea is to aggregate nodes hierarchically into areas: "close" nodes are aggregated into level 1 areas, "close" level 1 areas are aggregated into level 2 areas, and so on. An end-system maintains a view that contains the nodes in the same level 1 area, the level 1 areas in the same level 2 area, and so on. Thus an end-system maintains a smaller view than it would in the absence of hierarchy. Each area has its own QoS information derived from that of the subareas. A major problem of an area-based scheme is that aggregation results in loosing detailed link-level QoS information. This decreases the chance of the routing algorithm to choose "good" routes, i.e. routes that result in high successful VC setup rate (or equivalently high carried VC load).

\section{Our scheme}

In this paper, we present a scalable VC routing scheme that does not suffer from the problems of areas. Our scheme is based on the viewserver hierarchy we recently proposed in [3, 2] for large internetworks and evaluated for administrative policy constraints. Here, we are concerned with the support of performance/QoS requirements in large wide-area ATM-like networks, and we adapt our viewserver protocols accordingly.

In our scheme, views are not maintained by every end-system but by special switches called viewservers. For each viewserver, there is a subset of nodes around it, referred to as the viewserver's precinct. The viewserver only maintains the view of its precinct. This solves the scaling problem for storage requirement.

A viewserver can provide source routes for VCs between source and destination end-systems in its precinct. Obtaining a route between a source and a destination that are not in any single view involves accumulating the views of a sequence of viewservers. To make this process efficient, viewservers are organized hierarchically in levels, and an associated addressing structure is used. Each end-system has a set of addresses. Each address is a sequence of viewserver ids of decreasing levels, starting at the top level and going towards the end-system. The idea is that when the views of the viewservers in an address are merged, the merged view contains routes to the end-system 
from the top level viewservers.

We handle dynamic topology changes such as node/link failures and repairs, and link cost changes. Nodes detect topology changes affecting itself and neighbor nodes. Each node communicates these changes by flooding to the viewservers in a specified subset of nodes; this subset is referred to as its flood area. Hence, the number of packets used during flooding is proportional to the size of the flood area. This solves the scaling problem for the communication requirement.

Thus our VC routing protocol consists of two subprotocols: a view-query protocol between endsystems and viewservers for obtaining merged views; and a view-update protocol between nodes and viewservers for updating views.

\section{Evaluation}

In this paper, we compare our viewserver-based VC routing scheme to the simple scheme using VC-level simulation. In our simulation model, we define network topologies, QoS requirements, viewserver hierarchies, and evaluation measures. To the best of our knowledge, this is the first evaluation of a dynamic hierarchical-based VC routing scheme under real-time workload.

Our evaluation measures are the amount of memory required at the end-systems, the amount of time needed to construct a path ${ }^{2}$, the carried VC load, and the VC blocking probability. We use network topologies each of size 2764 nodes. Our results indicate that our viewserver-based VC routing scheme performs close to or better than the simple scheme in terms of VC carried load and blocking probability over a wide range of workload. It also reduces the amount of memory requirement by up to two order of magnitude.

\section{Organization of the paper}

In Section 2, we survey recent approaches to VC routing. In Section 3, we present the view-query protocol for static network conditions, that is, assuming all links and nodes of the network remain operational. In Section 4, we present the view-update protocol to handle topology changes. In Section 5, we present our evaluation model. Our results are presented in Section 6. Section 7 concludes the paper.

\footnotetext{
2 We use the terms route and path interchangeably.
} 


\section{Related Work}

In this section, we discuss routing protocols recently proposed for packet-switched QoS networks. These routing protocols can be classified depending on whether they help the network support qualitative QoS or quantitative (real-time) QoS. For a qualitative QoS, the network tries to provide the service requested by the application with no performance guarantees. Such a service is often identified as "best-effort". A quantitative QoS provides performance guarantees (typically required by real-time applications); for example, an upper bound on the end-to-end delay for any packet received at the destination.

Routing protocols that make routing decisions on a per $\mathrm{VC}$ basis can be used to provide either qualitative or quantitative QoS. For a quantitative QoS, some admission control tests should be performed during the VC-setup message's trip to the destination to try to reserve resources along the VC's path as described in Section 1.

On the other hand, the use of routing protocols that make routing decisions on a per packet basis is problematic in providing resource guarantees [5], and qualitative QoS is the best service the network can offer.

Since we are concerned in this paper with real-time QoS, we limit our following discussion to VC routing schemes proposed or evaluated in this context. We refer the reader to $[19,6]$ for a good survey on many other routing schemes.

Most of the VC routing schemes proposed for real-time QoS networks are based on the linkstate full-view approach described in Section $1[6,1,10,24]$. Recall that in this approach, each end-system maintains a view of the whole network, i.e. a graph with a vertex for every node and an edge between two neighbor nodes. QoS information is attached to the vertices and the edges of the view. This QoS information is distributed regularly to all end-systems to update their views and thus enable the selection of appropriate source routes for VCs, i.e. routes that are likely to meet the requested QoS. The proposed schemes mainly differ in how this QoS information is used. Generally, a cost function is defined in terms of the QoS information, and used to estimate the cost of a path to the VC's destination. The route selection algorithm then favors short paths with minimum cost. See $[17,22]$ for an evaluation of several schemes.

A number of VC routing schemes have also been designed for networks using the Virtual Path (VP) concept $[15,14]$. This VP concept has been proposed to simplify network management and control by having separate (logically) fully-connected subnetworks, typically one for each service class. In each VP subnetwork, simple routing schemes that only consider one-hop and two-hop 
paths are used. However, the advantage of using VPs can be offset by a decrease in statistical multiplexing gains of the subnetworks [15]. In this work, we are interested in general network topologies, where the shortest paths can be of arbitrary hop length and the overhead of routing protocols is of much concern.

All the above VC routing schemes are based on the link-state approach. VC routing schemes based on the path-vector approach have also been proposed [13]. In this approach, for each destination a node maintains a set of paths, one through each of its neighbor nodes. QoS information is attached to these paths. For each destination, a node exchanges its best feasible path ${ }^{3}$ with its neighbor nodes. The scheme in [13] provides two kinds of routes: pre-computed and on-demand. Pre-computed routes match some well-known QoS requirements, and are maintained using the path-vector approach. On-demand routes are calculated for specific QoS requirements upon request. In this calculation, the source broadcasts a special packet over all candidate paths. The destination then selects a feasible path from them and informs the source $[13,23]$. One drawback of this scheme is that obtaining on-demand routes is very expensive since there are potentially exponential number of candidate paths between the source and the destination.

The link-state approach is often proposed and favored over the path-vector approach in QoS architectures for several reasons [16]. An obvious reason is simplicity and complete control of the source over QoS route selection.

The above VC routing schemes do not scale well to large QoS networks in terms of storage and communication requirements. Several techniques to achieve scaling exist. The most common technique is the area hierarchy described in Section 1.

The landmark hierarchy $[26,25]$ is another approach for solving the scaling problem. The linkstate approach can not be used with the landmark hierarchy. A thorough study of enforcing QoS and policy constraints with this hierarchy has not been done.

Finally, we should point out that extensive effort is currently underway to fully specify and standardize VC routing schemes for the future integrated services Internet and ATM networks [9].

\section{Viewserver Hierarchy Query Protocol}

In this section, we present our scheme for static network conditions, that is, all links and nodes remain operational. The dynamic case is presented in Section 4.

\footnotetext{
${ }^{3}$ A feasible path is a path that satisfies the QoS constraints of the nodes in the path.
} 
Conventions: Each node has a unique id. NodeIds denotes the set of node-ids. For a node $u$, we use nodeid $(u)$ to denote the id of $u$. NodeNeighbors $(u)$ denotes the set of ids of the neighbors of $u$.

In our protocol, a node $u$ uses two kinds of sends. The first kind has the form "Send $(m)$ to $v$ ", where $m$ is the message being sent and $v$ is the destination-id. Here, nodes $u$ and $v$ are neighbors, and the message is sent over the physical link $(u, v)$. If the link is down, we assume that the packet is dropped.

The second kind of send has the form "Send $(m)$ to $v$ using $s r$ ", where $m$ and $v$ are as above and $s r$ is a source route between $u$ and $v$. We assume that as long as there is a sequence of up links connecting the nodes in $s r$, the message is delivered to $v$. This requires a transport protocol support such as TCP [20].

To implement both kind of sends, we assume there is a reserved VC on each link for sending routing, signaling and control messages [4]. This also ensures that routing messages do not degrade the QoS seen by applications.

\section{Views and Viewservers}

Views are maintained by special nodes called viewservers. Each viewserver has a precinct, which is a set of nodes around the viewserver. A viewserver maintains a view, consisting of the nodes in its precinct, links between these nodes and links outgoing from the precinct ${ }^{4}$. Formally, a viewserver $x$ maintains the following:

Precinct $_{x} \subseteq$ NodeIds. Nodes whose view is maintained.

View $w_{x}$. View of $x$.

$$
\begin{aligned}
& =\{\langle u, \text { timestamp, expirytime, }\{\langle v, \text { cost }\rangle: v \in \operatorname{NodeNeighbors}(u)\}\rangle: \\
& \left.u \in \text { Precinct }_{x}\right\}
\end{aligned}
$$

The intention of View $w_{x}$ is to obtain source routes between nodes in Precinct $t_{x}$. Hence, the choice of nodes to include in Precinct $t_{x}$ and the choice of links to include in View $w_{x}$ are not arbitrary. Precinct $_{x}$ and View Vust be connected; that is, between any two nodes in Precinct $_{x}$, there should be a path in View $w_{x}$. Note that View $w_{x}$ can contain links to nodes outside Precinct $t_{x}$. We say that a node $u$ is in the view of a viewserver $x$, if either $u$ is in the precinct of $x$, or View $w_{x}$ has a link from a node in the precinct of $x$ to node $u$. Note that the precincts and views of different viewservers can be overlapping, identical or disjoint.

\footnotetext{
4 Not all the links need to be included.
} 
For a link $(u, v)$ in the view of a viewserver $x, V i e w_{x}$ stores a cost. The cost of the link $(u, v)$ equals a vector of values if the link is known to be up; each cost value estimates how expensive it is to cross the link according to some QoS criteria such as delay, throughput, loss rate, etc. The cost equals $\infty$ if the link is known to be down. Cost of a link changes with time (see Section 4). The view also includes timestamp and expirytime fields which are described in Section 4.

\section{Viewserver Hierarchy}

For scaling reasons, we cannot have one large view. Thus, obtaining a source route between a source and a destination which are far away, involves accumulating views of a sequence of viewservers. To keep this process efficient, we organize viewservers hierarchically. More precisely, each viewserver is assigned a hierarchy level from $0,1, \ldots$, with 0 being the top level in the hierarchy. A parent-child relationship between viewservers is defined as follows:

1. Every level $i$ viewserver, $i>0$, has a parent viewserver whose level is less than $i$.

2. If viewserver $x$ is a parent of viewserver $y$ then $x$ 's precinct contains $y$ and $y$ 's precinct contains $x$.

3. The precinct of a top level viewserver contains all other top level viewservers.

In the hierarchy, a parent can have many children and a child can have many parents. We extend the range of the parent-child relationship to ordinary nodes; that is, if Precinct $t_{x}$ contains the node $u$, we say that $u$ is a child of $x$, and $x$ is a parent of $u$. We assume that there is at least one parent viewserver for each node.

For a node $u$, an address is defined to be a sequence $\left\langle x_{0}, x_{1}, \ldots, x_{t}\right\rangle$ such that $x_{i}$ for $i<t$ is a viewserver-id, $x_{0}$ is a top level viewserver-id, $x_{t}$ is the id of $u$, and $x_{i}$ is a parent of $x_{i+1}$. A node may have many addresses since the parent-child relationship is many-to-many. If a source node wants to establish a VC to a destination node, it first queries the name servers to obtain a set of addresses for the destination ${ }^{5}$. Second, it queries viewservers to obtain an accumulated view containing both itself and the destination node (it can reach its parent viewservers by using fixed source routes to them). Then, it chooses a feasible source route from this accumulated view and initiates the VC setup protocol on this path.

\section{View-Query Protocol: Obtaining Source Routes}

We now describe how a source route is obtained.

\footnotetext{
5 Querying the name servers can be done in the same way as is done currently in the Internet.
} 
We want a sequence of viewservers whose merged views contains both the source and the destination nodes. Addresses provide a way to obtain such a sequence, by first going up in the viewserver hierarchy starting from the source node and then going down in the viewserver hierarchy towards the destination node. More precisely, let $\left\langle s_{0}, \ldots, s_{t}\right\rangle$ be an address of the source, and $\left\langle d_{0}, \ldots, d_{l}\right\rangle$ be an address of the destination. Then, the sequence $\left\langle s_{t-1}, \ldots, s_{0}, d_{0}, \ldots, d_{l-1}\right\rangle$ meets our requirements. In fact, going up all the way in the hierarchy to top level viewservers may not be necessary. We can stop going up at a viewserver $s_{i}$ if there is a viewserver $d_{j}, j<l$, in the view of $s_{i}$ (one special case is where $s_{i}=d_{j}$ ).

The view-query protocol uses two message types:

- (RequestView, s_address, d_address) where $s_{-} a d d r e s s$ and $d_{-} a d d r e s s$ are the addresses for the source and the destination respectively. A RequestView message is sent by a source node to obtain an accumulated view containing both the source and the destination nodes. When a viewserver receives a RequestView message, it either sends back its view or forwards this request to another viewserver.

- (ReplyView, s_address, d_address, accumview) where $s_{-}$address and $d_{-}$address are as above and accumview is the accumulated view. A ReplyView message is sent by a viewserver to the source or to another viewserver closer to the source. The accumview field in a Replyview message equals the union of the views of the viewservers the message has visited.

We now describe the view-query protocol in more detail (please refer to Figures 1 and 2). To establish a VC to a destination node, the source node sends a RequestView packet containing the source and the destination addresses to its parent in the source address.

Upon receiving a RequestView packet, a viewserver $x$ checks if the destination node is in its precinct $^{6}$. If it is, $x$ sends back its view in a ReplyView packet. If it is not, $x$ forwards the request packet to another viewserver as follows (details in Figure 2): $x$ checks whether any viewserver in the destination address is in its view. If there is such a viewserver, $x$ sends the RequestView packet to the last such one in the destination address. Otherwise $x$ is a viewserver in the source address, and it sends the packet to its parent in the source address.

When a viewserver $x$ receives a ReplyView packet, it merges its view to the accumulated view in the packet. Then it sends the Replyview packet towards the source node in the same way it would send a RequestView packet towards the destination node (i.e. the roles of the source address

\footnotetext{
${ }^{6}$ Even though the destination can be in the view of $x$, its QoS characteristics is not in the view if it is not in the precinct of $x$.
} 


\section{Constants}

FixedRoutes $s_{u}(x)$, for every viewserver-id $x$ such that $x$ is a parent of $u$, $=\left\{\left\langle y_{1}, \ldots, y_{n}\right\rangle: y_{i} \in\right.$ NodeIds $\}$. Set of routes to $x$

\section{Events}

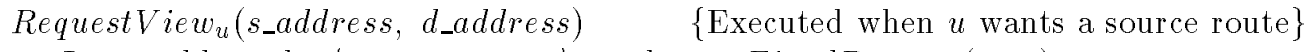

Let $s_{-}$address be $\left\langle s_{0}, \ldots, s_{t-1}, s_{t}\right\rangle$, and $s r \in$ FixedRoutes $s_{u}\left(s_{t-1}\right)$;

Send(RequestView, s_address, d_address) to $s_{t-1}$ using $s r$

Receive $_{u}$ (ReplyView, s_address, d_address, accumview)

Choose a feasible source route using accumview;

If a feasible route is not found

Execute RequestView $w_{u}$ again with another source address and/or destination address

Figure 1: View-query protocol: Events and state of a source node $u$.

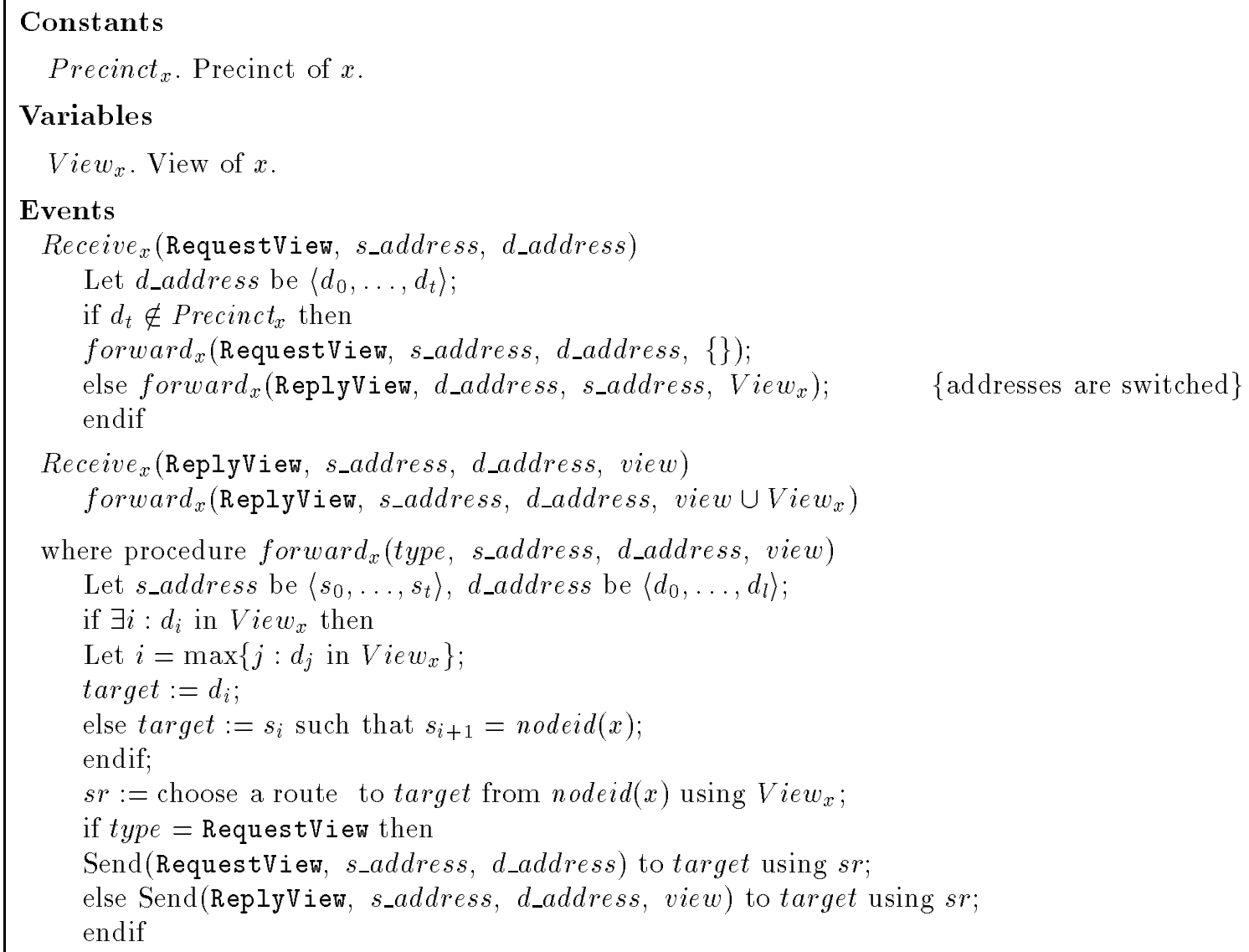

Precinct $_{x}$. Precinct of $x$.

\section{Variables}

View. View of $x$.

\section{Events}

Receive $_{x}$ (Requestview, s_address, d_address)

Let $d_{-}$address be $\left\langle d_{0}, \ldots, d_{t}\right\rangle$;

if $d_{t} \notin$ Precinct $_{x}$ then

forward $_{x}$ (RequestView, s_address, d_address, \{\}$)$;

else forward fReplyView, d_address, s_address, View $\left._{x}\right) ; \quad$ \{addresses are switched\} endif

Receive $_{x}($ Replyview, s_address, d_address, view)

forward $_{x}\left(\right.$ ReplyView, s_address, d_address, view $\cup$ View $w_{x}$ )

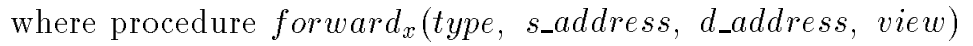

Let $s_{-}$address be $\left\langle s_{0}, \ldots, s_{t}\right\rangle$, d_address be $\left\langle d_{0}, \ldots, d_{l}\right\rangle$;

if $\exists i: d_{i}$ in $V i e w_{x}$ then

Let $i=\max \left\{j: d_{j}\right.$ in View $\left._{x}\right\}$;

target $:=d_{i}$;

else target $:=s_{i}$ such that $s_{i+1}=\operatorname{nodeid}(x)$;

endif;

$s r:=$ choose a route to target from nodeid( $x)$ using View $;$

if type = RequestView then

Send(RequestView, s_address, d_address) to target using sr;

else Send(ReplyView, s_address, d_address, view) to target using sr;

endif

Figure 2: View-query protocol: Events and state of a viewserver $x$.

and the destination address are interchanged). 
When the source receives a ReplyView packet, it chooses a feasible path using the accumview in the packet. If it does not find a feasible path, it can try again using a different source and/or destination addresses. Note that the source does not have to throw away the previous accumulated views; it can merge them all into a richer accumulated view. In fact, it is easy to change the protocol so that the source can also obtain views of individual viewservers to make the accumulated view even richer. Once a feasible source route is found, the source node initiates the VC setup protocol.

Above we have described one possible way of obtaining the accumulated views. There are various other possibilities, for example: (1) restricting the ReplyView packet to take the reverse of the path that the RequestView packet took; (2) having ReplyView packets go all the way up in the viewserver-hierarchy for a richer accumulated view; (3) having the source poll the viewservers directly instead of the viewservers forwarding request/reply messages to each other; (4) not including non-transit nodes (e.g. end-systems) other than the source and the destination nodes in the accumview; (5) including some QoS requirements in the Requestview packet, and having the viewservers filter out some nodes and links.

\section{Update Protocol for Dynamic Network Conditions}

In this section, we first describe how topology changes such as link/node failures, repairs and cost changes, are detected and communicated to viewservers, i.e. the view-update protocol. Then, we modify the view-query protocol appropriately.

\section{View-Update Protocol: Updating Views}

Viewservers do not communicate with each other to maintain their views. Nodes detect and communicate topology changes to viewservers. Updates are done periodically and also optionally after a change in the outgoing link costs.

The communication between a node and viewservers is done by flooding over a set of nodes. This set is referred to as the flood area. The topology of a flood area must be a connected graph. For efficiency, the flood area can be implemented by a hop-count.

Due to the nature of flooding, a viewserver can receive information out of order from a node. In order to avoid old information replacing new information, each node includes successively increasing time stamps in the messages it sends. The timestamp field in the view of a viewserver equals the largest timestamp received from each node. 
Due to node and link failures, communication between a node and a viewserver can fail, resulting in the viewserver having out-of-date information. To eliminate such information, a viewserver deletes any information about a node if it is older than a time-to-die period. The expirytime field in the view of a viewserver equals the end of the time-to-die period for a node. We assume that nodes send messages more often than the time-to-die value (to avoid false removal).

The view-update protocol uses one type of message as follows:

- (Update, nid, timestamp, floodarea, ncostset) is sent by the node to inform the viewservers about current costs of its outgoing links. Here, nid and timestamp indicate the id and the time stamp of the node, ncostset contains a cost for each outgoing link of the node, and floodarea is the set of nodes that this message is to be sent over.

\section{Constants:}

FloodAreag. ( $\subseteq$ NodeIds). The flood area of the node.

\section{Variables:}

Clock : Integer. Clock of $g$.

Figure 3: State of a node $g$.

The state maintained by a node $g$ is listed in Figure 3. We assume that consecutive reads of Clock $_{g}$ returns increasing values.

\section{Constants:}

Precinct $x$. Precinct of $x$.

TimeToDie $_{x}$ : Integer. Time-to-die value.

\section{Variables:}

Vie $w_{x}$. View of $x$.

Clock : Integer. Clock of $x$.

Figure 4: State of a viewserver $x$.

The state maintained by a viewserver $x$ is listed in Figure 4.

The events of node $g$ are specified in Figure 5. The events of a viewserver $x$ are specified in Figure 6 . When a viewserver $x$ recovers, View $w_{x}$ is set to \{\} . Its view becomes up-to-date as it receives new information from nodes (and remove false information with the time-to-die period). 


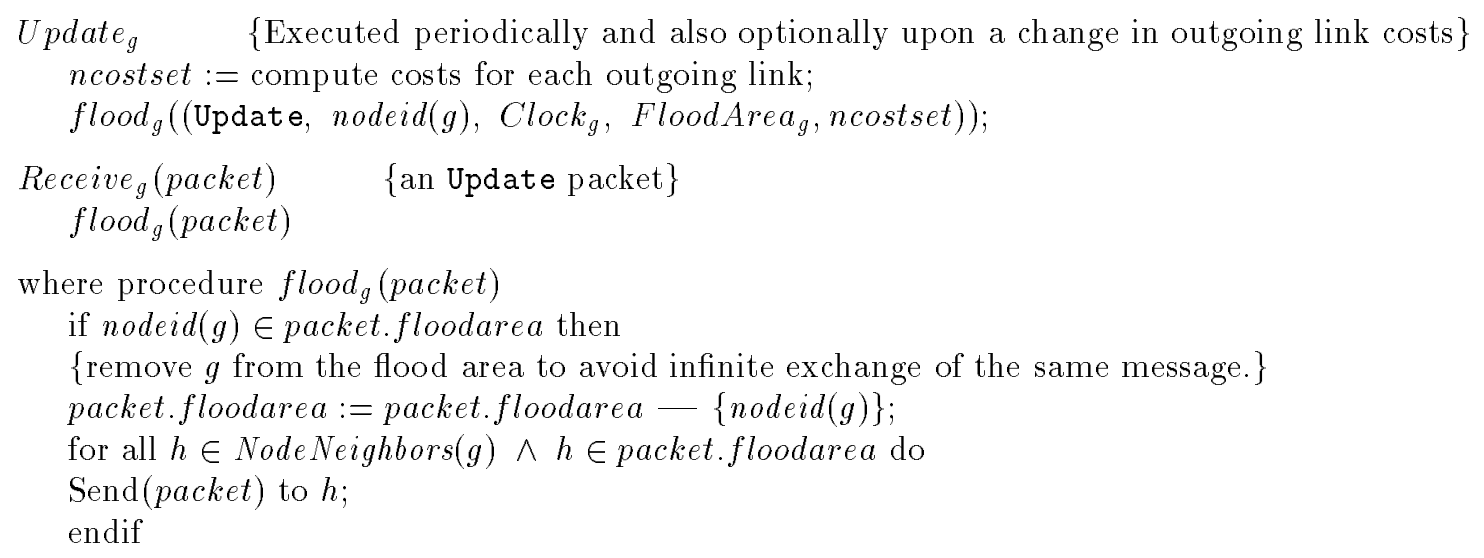

Node Failure Model: A node can undergo failures and recoveries at anytime. We assume failures are fail-stop (i.e. a failed node does not send erroneous messages).

Figure 5: View-update protocol: Events of a node $g$.

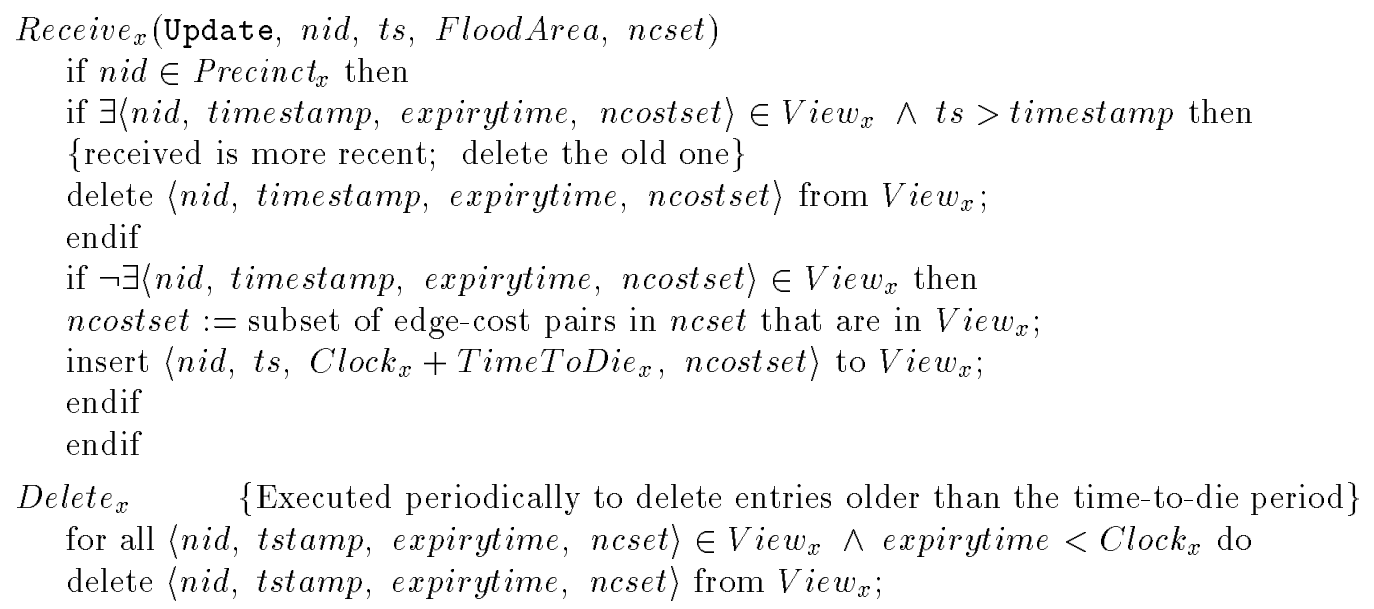

Viewserver Failure Model: A viewserver can undergo failures and recoveries at anytime. We assume failures are fail-stop. When a viewserver $x$ recovers, View $w_{x}$ is set to \{\} .

Figure 6: View update events of a viewserver $x$.

\section{Changes to View-Query Protocol}

We now enumerate the changes needed to adapt the view-query protocol to the dynamic case (the formal specification is omitted for space reasons).

Due to link and node failures, RequestView and ReplyView packets can get lost. Hence, the 
source may never receive a ReplyView packet after it initiates a request. Thus, the source should try again after a time-out period.

When a viewserver receives a RequestView message, it should reply with its views only if the destination node is in its precinct and its view contains a path to the destination. Similarly during forwarding of RequestView and ReplyView packets, a viewserver, when checking whether a node is in its view, should also check if its view contains a path to it.

\section{Evaluation}

In this section, we present the parameters of our simulation model. We use this model to compare our viewserver-based VC routing protocols to the simple approach. The results obtained are presented in Section 6.

\section{Network Parameters}

We model a campus network which consists of a campus backbone subnetwork and several department subnetworks. The backbone network consists of backbone switches and backbone links.

Each department network consists of a hub switch and several non-hub switches. Each non-hub switch has a link to the department's hub switch. And the department's hub switch has a link to one of the backbone switches. A non-hub switch can have links to other non-hub switches in the same department, to non-hub switches in other departments, or to backbone switches.

End-systems are connected to non-hub switches. An example network topology is shown in Figure 7.

In our topology, there are 8 backbone switches and 32 backbone links. There are 16 departments. There is one hub-switch in each department. There is a total of 240 non-hub switches randomly assigned to different departments. There are 2500 end-systems which are randomly connected to non-hub switches. Thus, we have a total of 2764 nodes.

In addition to the links connecting non-hub switches to the hub switches and hub switches to the backbone switches, there are 720 links from non-hub switches to non-hub switches in the same department, there are 128 links from non-hub switches to non-hub switches in different departments, and there are 64 links from non-hub switches to backbone switches.

The end-points of each link are chosen randomly. However, we make sure that the backbone network is connected; and there is a link from node $u$ to node $v$ iff there is a link from node $v$ to 


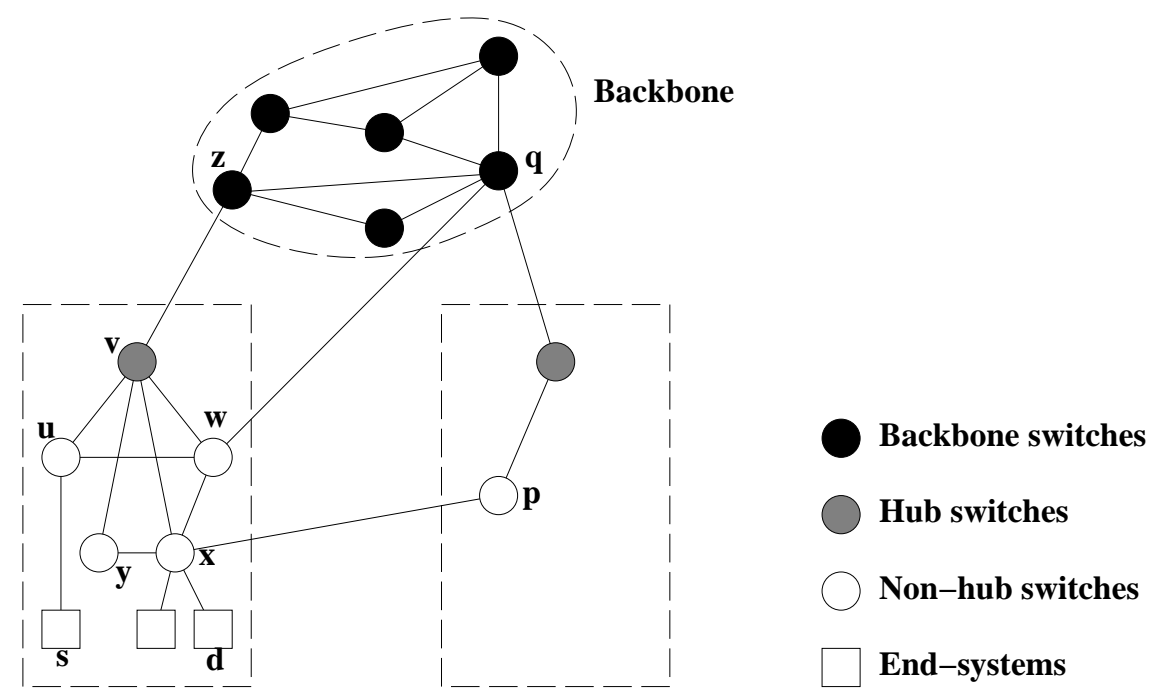

Department 1 Department 2

Figure 7: An example network topology.

node $u$.

Each link has a total of $C$ units of bandwidth.

\section{QoS and Workload Parameters}

In our evaluation model, we assume that a VC requires the reservation of a certain amount of bandwidth that is enough to ensure an acceptable QoS for the application. This reservation amount can be thought of either as the peak transmission rate of the VC or its "effective bandwidth" [12] varying between the peak and average transmission rate.

VC setup requests arrive to the network according to a Poisson process of rate $\lambda$, each requiring one unit of bandwidth. Each VC, once it is successfully setup, has a lifetime of exponential duration with mean $1 / \mu$. The source and the destination end-systems of a VC are chosen randomly.

An arriving $\mathrm{VC}$ is admitted to the network if at least one feasible path between its source and destination end-systems is found by the routing protocol, where a feasible path is one that has links with non-zero available capacity. From the set of feasible paths, a minimum hop path is used to establish the VC; one unit of bandwidth is allocated on each of its links for the lifetime of the VC. On the other hand, if a feasible path is not found, then the arriving VC is blocked and lost.

We assume that the available link capacities in the views of the viewservers are updated instan- 
taneously whenever a VC is admitted to the network or terminates.

\section{Viewserver Hierarchy Schemes}

We have evaluated our viewserver protocol for several different viewserver hierarchies and query methods. We next describe the different viewserver schemes evaluated. Please refer to Figure 7 in the following discussion.

The first viewserver scheme is referred to as base. Each switch is a viewserver. A viewserver's precinct consist of itself and the neighboring nodes. The links in the viewserver's view consist of the links between the nodes in the precinct, and links outgoing from nodes in the precinct to nodes not in the precinct. For example, the precinct of viewserver $u$ consists of nodes $u, v, w, s$.

As for the viewserver hierarchy, a backbone switch is a level 0 viewserver, a hub switch is a level 1 viewserver and a non-hub switch is a level 2 viewserver. Parent of a hub switch viewserver is the backbone switch viewserver it is connected to. Parent of a non-hub switch viewserver is the hub switch viewserver in its department. Parent of an end-system is the non-hub switch viewserver it is connected to.

We use only one address for each end-system. The viewserver-address of an end-system is the concatenation of four ids. Thus, the address of $s$ is z.v.u.s. Similarly, the address of $d$ is z.v.x.d. To obtain a route between $s$ and $d$, it suffices to obtain views of viewservers $u, v, x$.

The second viewserver scheme is referred to as base-QT (where the $Q T$ stands for "query up to top"). It is identical to base except that during the query protocol all the viewservers in the source and the destination addresses are queried. That is, to obtain a route between $s$ and $d$, the views of $u, v, x, z$ are obtained.

The third viewserver scheme is referred to as vertex-extension. It is identical to base except that viewserver precincts are extended as follows: Let $\mathrm{P}$ denote the precinct of a viewserver in the base scheme. For each node $u$ in $\mathrm{P}$, if there is a link from node $u$ to node $v$ and $v$ is not in $\mathrm{P}$, node $v$ is added to the precinct; among $v$ 's links, only the ones to nodes in $\mathrm{P}$ are added to the view. In the example, nodes $z, y, x, q$ are added to the precinct of $u$, but outgoing links of these nodes to other nodes are not included (e.g. $(x, p)$ and $(z, q)$ are not included). The advantage of this scheme is that even though it increases the precinct size by a factor of $d$ (where $d$ is the average number of neighbors to a node), it increases the number of links stored in the view by a factor less than 2 .

The fourth viewserver scheme is referred to as vertex-extension-QT. It is identical to vertexextension except that during the query protocol all the viewservers in the source and the destination 
addresses are queried.

\section{Numerical Results}

\subsection{Results for Network 1}

The parameters of the first network topology, referred to as Network 1, are given in Section 5. The link capacity $C$ is taken to be 20 [6], i.e. a link is capable of carrying 20 VCs simultaneously.

Our evaluation measures were computed for a (randomly chosen but fixed) set of 100,000 VC setup requests. Table 1 lists for each viewserver scheme (1) the minimum, average and maximum of the precinct sizes (in number of nodes), (2) the minimum, average and maximum of the merged view sizes (in number of nodes), and (3) the minimum, average and maximum of the number of viewservers queried.

\begin{tabular}{|l||c|c|c|}
\hline Scheme & Precinct Size & Merged View Size & No. of Viewservers Queried \\
\hline base & $5 / 16.32 / 28$ & $4 / 56.46 / 81$ & $1 / 5.49 / 6$ \\
\hline base- $Q T$ & $5 / 16.32 / 28$ & $27 / 59.96 / 81$ & $6 / 6.00 / 6$ \\
\hline vertex-extension & $22 / 88.11 / 288$ & $14 / 155.86 / 199$ & $1 / 5.49 / 6$ \\
\hline vertex-extension- $Q T$ & $22 / 88.11 / 288$ & $113 / 163.28 / 199$ & $6 / 6.00 / 6$ \\
\hline
\end{tabular}

Table 1: Precinct sizes, merged view sizes, and number of viewservers queried for Network 1.

The precinct size indicates the memory requirement at a viewserver. More precisely, the memory requirement at a viewserver is $O$ (precinct size $\times d$ ), except for the vertex-extension and vertexextension- $Q T$ schemes. In these schemes, the memory requirement is increased by a factor less than two. Hence these schemes have the same order of viewserver memory requirement as the base and base-QT schemes.

The merged view size indicates the memory requirement at a source end-system during the query protocol; i.e. the memory requirement at a source end-system is $O($ merged view size $\times d)$ except for the vertex-extension and vertex-extension-QT schemes. Note that the source end-system does not need to store information about end-systems other than itself and the destination. The numbers in Table 1 take advantage of this.

The number of viewservers queried indicates the communication time required to obtain the merged view at the source end-system. Hence, the "real-time" communication time required to obtain the merged view at a source is slightly more than one round-trip time between the source 
and the destination.

As is apparent from Table 1, using a $Q T$ scheme increases the merged view size by about $6 \%$, and the number of viewservers queried by about $9 \%$. Using the vertex-extension scheme increases the merged view size by about 3 times (note that the amount of actual memory needed increases only by a factor less than 2).

The above measures show the memory and time requirements of our protocols. They clearly indicate the savings in storage over the simple approach as manifested by the smaller view sizes. To answer whether the viewserver hierarchy finds many feasible paths, other evaluation measures such as the carried VC load and the percent VC blocking are of interest. They are defined as follows:

- Carried VC load is the average number of VCs carried by the network.

- Percent VC blocking is the percentage of VC setup requests that are blocked due to the fact that a feasible path is not found. ${ }^{7}$

In our experiments, we keep the average VC lifetime $(1 / \mu)$ fixed at 15000 and vary the arrival rate of VC setup requests $(\lambda)$. Figure 8 shows the carried VC load versus $\lambda$ for the simple approach and the viewserver schemes. Figure 9 shows the percent $\mathrm{VC}$ blocking versus $\lambda$. At low values of $\lambda$, all the viewserver schemes are very close to the simple approach. At moderate values of $\lambda$, the base and base-QT schemes perform badly. The vertex-extension and vertex-extension-QT schemes are still very close to the simple approach (only $3.4 \%$ less carried VC load). Note that the performance of the viewserver schemes can be further improved by trying more viewserver addresses.

Surprisingly, at high values of $\lambda$, all the viewserver schemes perform better than the simple approach. At $\lambda=0.5$, the network with the base scheme carries about $30 \%$ higher load than the simple approach. This is an interesting result. Our explanation is as follows. Elsewhere [2], we have found that when the viewserver schemes can not find an existing feasible path, this path is usually very long (more than 11 hops). This causes our viewserver hierarchy protocols to reject VCs that are admitted by the simple approach over long paths. The use of long paths for VCs is undesirable since it ties up resources at more intermediate nodes, which can be used to admit many shorter length VCs.

In conclusion, we recommend the vertex-extension scheme as it performs close to or better than all other schemes in terms of VC carried load and blocking probability over a wide range of workload. Note that for all viewserver schemes, adding $Q T$ yields slightly further improvement.

\footnotetext{
7 Recall that we assume a blocked VC setup request is cleared (i.e. lost).
} 


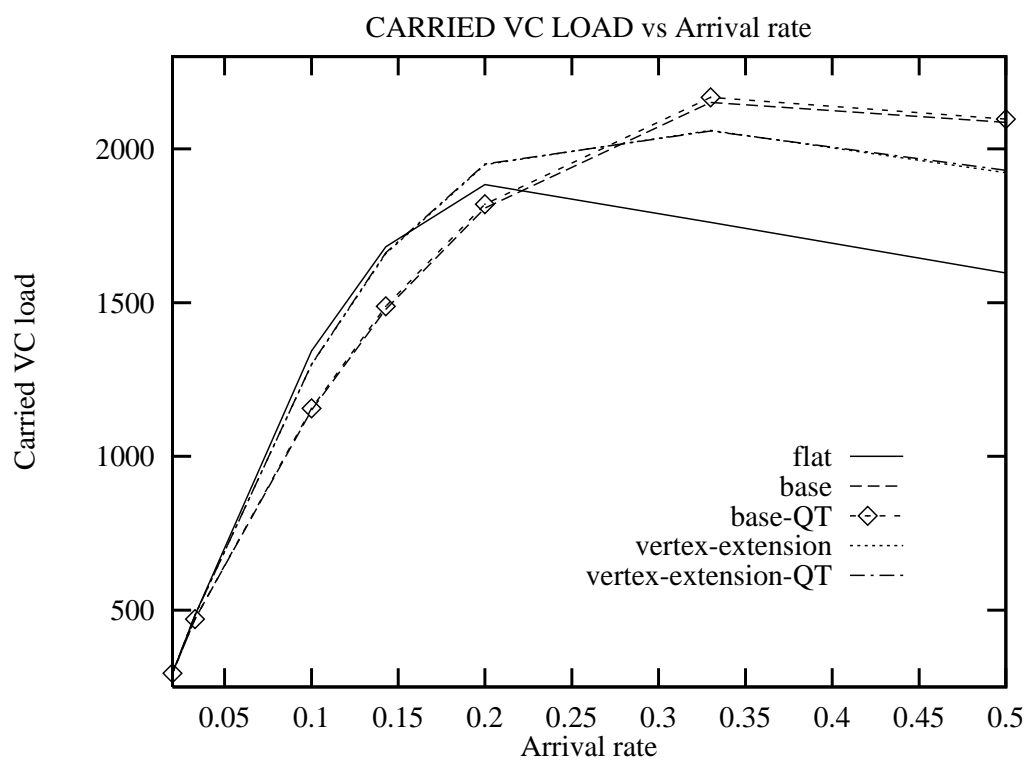

Figure 8: Carried VC load versus arrival rate for Network 1.

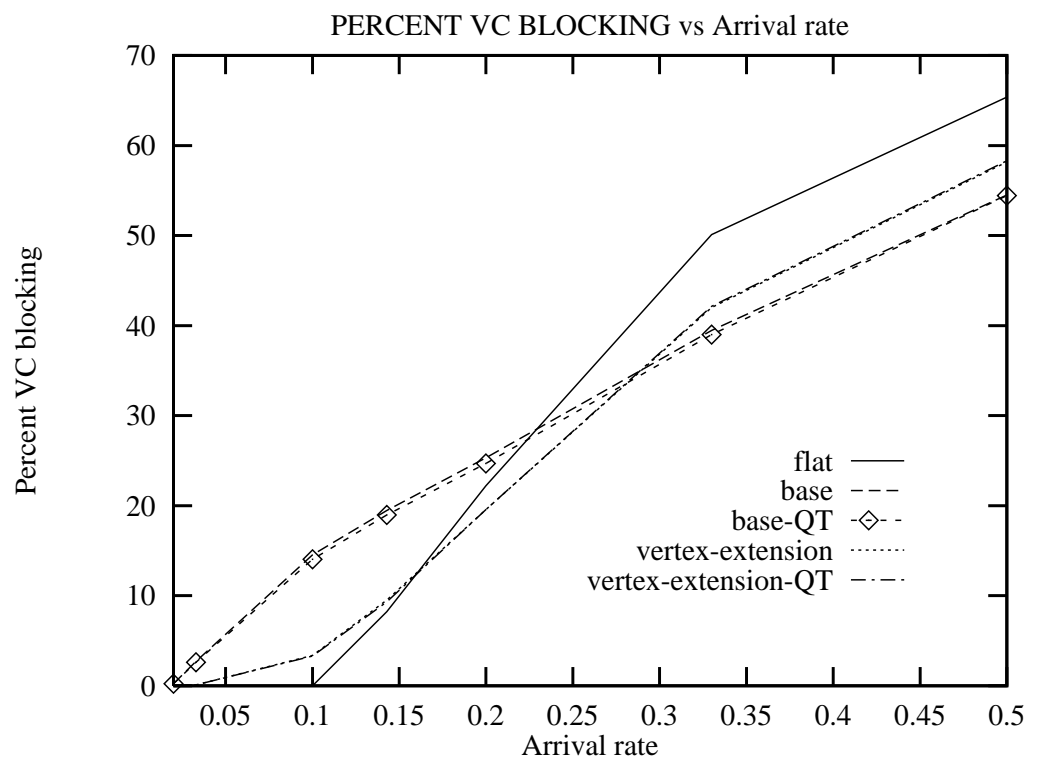

Figure 9: Percent VC blocking versus arrival rate for Network 1.

\subsection{Results for Network 2}

The parameters of the second network, referred to as Network 2, are the same as the parameters of Network 1. However, a different seed is used for the random number generation, resulting in a different topology and distribution of source-destination end-system pairs for the VCs.

We again take $C=20$, and we fix $1 / \mu$ at 15000 . Our evaluation measures were computed for 
a set of 100,000 VC setup requests. Table 2, and Figures 10 and 11 show the results. Similar conclusions to Network 1 hold for Network 2. An interesting exception is that at high values of $\lambda$, we observe that the vertex-extension scheme performs slightly better than the vertex-extension- $Q T$ scheme (about 4.2\% higher carried VC load). The reason is the following: Adding $Q T$ gives richer merged views, and hence increases the chance of finding a feasible path that is possibly long. As explained in Section 6.1, this results in performance degradation.

\begin{tabular}{|l||c|c|c|}
\hline Scheme & Precinct Size & Merged View Size & No. of Viewservers Queried \\
\hline base & $4 / 16.32 / 33$ & $4 / 57.61 / 80$ & $1 / 5.52 / 6$ \\
\hline base-QT & $4 / 16.32 / 33$ & $30 / 60.64 / 80$ & $6 / 6.00 / 6$ \\
\hline vertex-extension & $17 / 90.36 / 282$ & $16 / 159.70 / 214$ & $1 / 5.52 / 6$ \\
\hline vertex-extension-QT & $17 / 90.36 / 282$ & $113 / 166.97 / 214$ & $6 / 6.00 / 6$ \\
\hline
\end{tabular}

Table 2: Precinct sizes, merged view sizes, and number of viewservers queried for Network 2.

We have repeated the above evaluations for other networks and obtained similar conclusions.

\section{Conclusions}

We presented a hierarchical VC routing protocol for ATM-like networks. Our protocol satisfies QoS constraints, adapts to dynamic topology changes, and scales well to large number of nodes.

Our protocol uses partial views maintained by viewservers. The viewservers are organized hierarchically. To setup a VC, the source end-system queries viewservers to obtain a merged view that contains itself and the destination end-system. This merged view is then used to compute a source route for the $\mathrm{VC}$.

We evaluated several viewserver hierarchy schemes and compared them to the simple approach. Our results on 2764-node networks indicate that the vertex-extension scheme performs close to or better than the simple approach in terms of VC carried load and blocking probability over a wide range of real-time workload. It also reduces the amount of memory requirement by up to two order of magnitude. We note that our protocol scales even better on larger size networks [3].

In all the viewserver schemes we studied, each switch is a viewserver. In practice, not all switches need to be viewservers. We may associate one viewserver with a group of switches. This is particularly attractive in ATM networks where each signaling entity is responsible for establishing VCs across a group of nodes. In such an environment, viewservers and signaling entities can be 


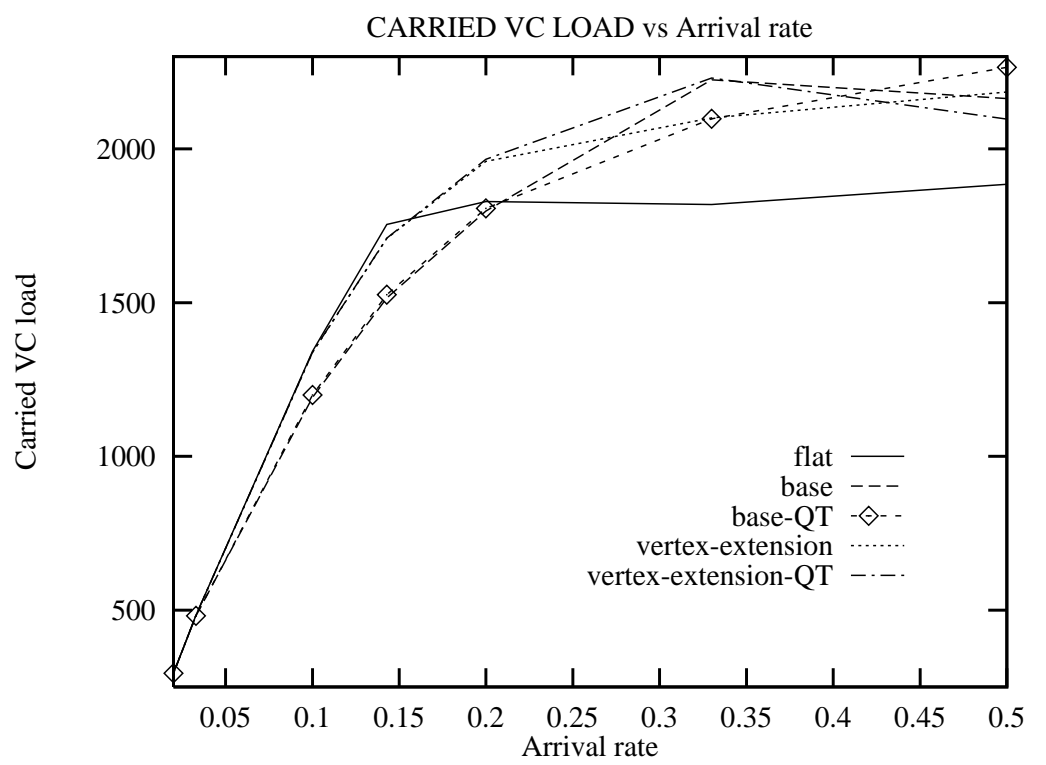

Figure 10: Carried VC load versus arrival rate for Network 2.

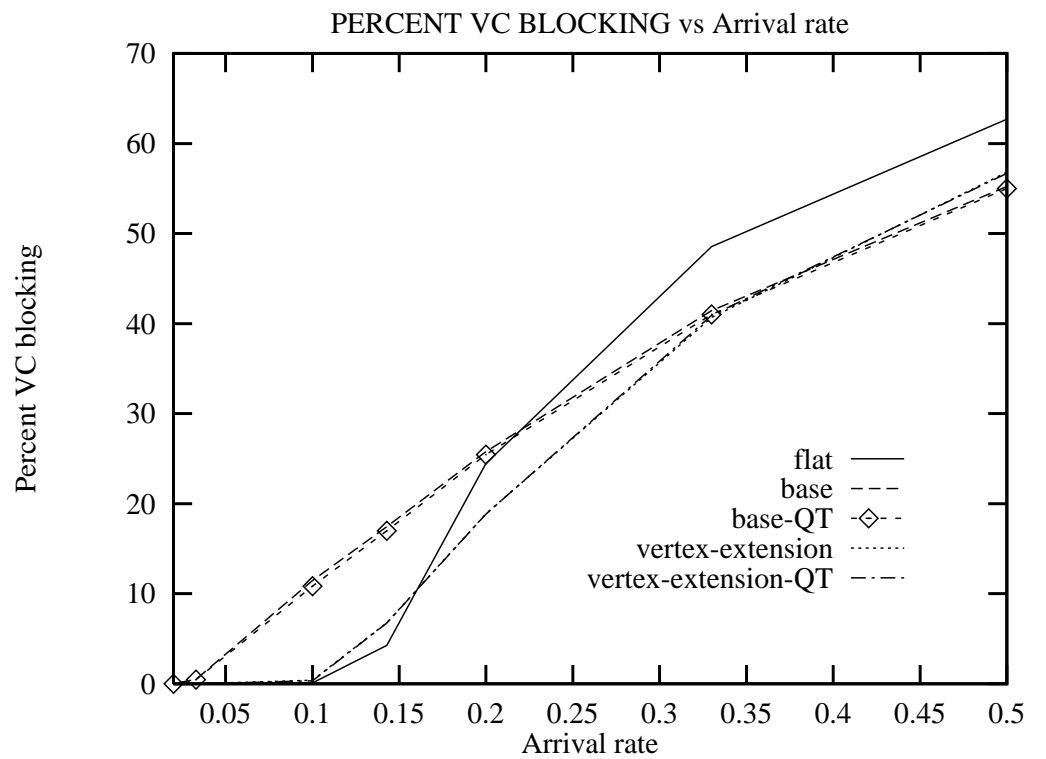

Figure 11: Percent VC blocking versus arrival rate for Network 2.

combined.

However, there is an advantage of each switch being a viewserver; that is, source nodes do not require fixed source routes to their parent viewservers (in the view-query protocol). This reduces the amount of hand configuration required. In fact, the base and base-QT viewserver schemes do not require any hand configuration.

Our evaluation model assumed that views are instantaneously updated, i.e. no delayed feedback 
between link cost changes and view/route changes. We plan to investigate the effect of delayed feedback on the performance of the different schemes. We expect our viewserver schemes to outperform the simple approach in this realistic setting as the update of views of the viewservers requires less time and communication overhead. Thus, views in our viewserver schemes will be more up-to-date.

As we pointed out in [3], the only drawback of our protocol is that to obtain a source route for a VC, views are merged at (or prior to) the VC setup, thereby increasing the setup time. This drawback is not unique to our scheme $[8,16,7,11]$. Reference [3] describes several ways, including cacheing and replication, to reduce the setup overhead and improve performance.

\section{References}

[1] H. Ahmadi, J. Chen, and R. Guerin. Dynamic Routing and Call Control in High-Speed Integrated Networks. In Proc. Workshop on Systems Engineering and Traffic Engineering, ITC'13, pages 19-26, Copenhagen, Denmark, June 1991.

[2] C. Alaettinoğlu and A. U. Shankar. Viewserver Hierarchy: A New Inter-Domain Routing Protocol and its Evaluation. Technical Report UMIACS-TR-93-98, CS-TR-3151, Department of Computer Science, University of Maryland, College Park, October 1993. Earlier version CS-TR-3033, February 1993.

[3] C. Alaettinoğlu and A. U. Shankar. Viewserver Hierarchy: A New Inter-Domain Routing Protocol. In Proc. IEEE INFOCOM'94, Toronto, Canada, June 1994.

[4] A. Alles. ATM in Private Networking: A Tutorial. Hughes LAN Systems, 1993.

[5] P. Almquist. Type of Service in the Internet Protocol Suite. Technical Report RFC-1349, Network Working Group, July 1992.

[6] L. Breslau, D. Estrin, and L. Zhang. A Simulation Study of Adaptive Source Routing in Integrated Services Networks. Available by anonymous ftp at catarina.usc.edu:pub/breslau, September 1993.

[7] J. N. Chiappa. A New IP Routing and Addressing Architecture. Big-Internet mailing list., 1992. Available by anonymous ftp from munnari.oz .au:big-internet/list-archive.

[8] D.D. Clark. Policy routing in Internet protocols. Request for Comment RFC-1102, Network Information Center, May 1989.

[9] R. Coltun and M. Sosa. VC Routing Criteria. Internet Draft, March 1993.

[10] D. Comer and R. Yavatkar. FLOWS: Performance Guarantees in Best Effort Delivery Systems. In Proc. IEEE INFOCOM, Ottawa, Canada, pages 100-109, April 1989.

[11] D. Estrin, Y. Rekhter, and S. Hotz. Scalable Inter-Domain Routing Architecture. In Proc. $A C M$ SIGCOMM'92, pages 40-52, Baltimore, Maryland, August 1992.

[12] R. Guerin, H. Ahmadi, and M. Naghshineh. Equivalent Capacity and its Application to Bandwidth Allocation in High-Speed Networks. IEEE J. Select. Areas Commun., SAC-9(7):968-981, September 1991.

[13] A. Guillen, R. Kia, and B. Sales. An Architecture for Virtual Circuit/QoS Routing. In Proc. IEEE International Conference on Network Protocols '93, pages 80-87, San Francisco, California, October 1993.

[14] S. Gupta, K. Ross, and M. ElZarki. Routing in Virtual Path Based ATM Networks. In Proc. GLOBECOM'g2, pages 571-575, 1992.

[15] R-H. Hwang, J. Kurose, and D. Towsley. MDP Routing in ATM Networks Using Virtual Path Concept. In Proc. IEEE INFOCOM, pages 1509-1517, Toronto, Ontario, Canada, June 1994.

[16] M. Lepp and M. Steenstrup. An Architecture for Inter-Domain Policy Routing. Internet Draft. Available from the authors., June 1992.

[17] I. Matta and A.U. Shankar. An Iterative Approach to Comprehensive Performance Evaluation of Integrated Services Networks. In Proc. IEEE International Conference on Network Protocols '94, Boston, Massachusetts, October 1994. To appear.

[18] J. Moy. OSPF Version 2. RFC 1247, Network Information Center, SRI International, July 1991. 
[19] C. Parris and D. Ferrari. A Dynamic Connection Management Scheme for Guaranteed Performance Services in Packet-Switching Integrated Services Networks. Technical Report TR-93-005, International Computer Science Institute, Berkeley, California, January 1993.

[20] J. Postel. Transmission Control Protocol: DARPA Internet Program Protocol Specification. Request for Comment RFC-793, Network Information Center, SRI International, 1981.

[21] M. Prycker. Asynchronous Transfer Mode - Solution for Broadband ISDN. Ellis Horwood, 1991.

[22] S. Rampal, D. Reeves, and D. Agrawal. An Evaluation of Routing and Admission Control Algorithms for Multimedia Traffic in Packet-Switched Networks. Available from the authors, 1994.

[23] H. Suzuki and F. Tobagi. Fast Bandwidth Reservation Scheme with Multi-Link and Multi-Path Routing in ATM Networks. In Proc. IEEE INFOCOM '92, pages 2233-2240, Florence, Italy, May 1992.

[24] E. Sykas, K. Vlakos, I. Venieris, and E. Protonotarios. Simulative Analysis of Optimal Resource Allocation and Routing in IBCN's. IEEE J. Select. Areas Commun., 9(3):486-492, April 1991.

[25] P. F. Tsuchiya. The Landmark Hierarchy: Description and Analysis, The Landmark Routing: Architecture Algorithms and Issues. Technical Report MTR-87W00152, MTR-87W00174, The MITRE Corporation, McLean, Virginia, 1987.

[26] P. F. Tsuchiya. The Landmark Hierarchy:A New Hierarchy For Routing In Very Large Networks. In Proc. ACM SIGCOMM'88, August 1988. 